\title{
A importância do uso de fitoterápicos como prática alternativa ou complementar na atenção básica: revisão da literatura
}

The importance of using herbal medicines as an alternative or complementary practice in primary care: literature review

La importancia del uso de las medicinas a base de hierbas como práctica alternativa o complementaria en atención primaria: revisión de la literatura

\section{Resumo}

As plantas medicinais vem sendo uma solução considerada nos problemas de saúde pública. A Organização Mundial de Saúde (OMS) ressalta que até $80 \%$ da população de países em desenvolvimento confiam na Medicina Complementar ou Alternativa (MAC) em cuidados iniciais a saúde. Dessa forma, A pesquisa tem como objetivo descrever a importância do uso de fitoterápicos como tratamento alternativo e complementar na atenção básica. $\mathrm{O}$ presente estudo trata-se de uma revisão integrativa da literatura com teor descritivo e de natureza qualitativa. Os dados para esse estudo foram obtidos nas bases de dados: Biblioteca Virtual em Saúde (BVS), Scielo, Lilacs, medline e no diretório Google Acadêmico. Foram considerados estudos originais disponíveis e publicados na íntegra que abordassem a questão norteadora, entre os anos de 2000 e 2020; nos idiomas português, inglês ou espanhol. Resultados: Visando uma maior segurança e maior controle na utilização de medicamentos fitoterápicos, a ANVISA (Agência Nacional de Vigilância Sanitária), dispõe de várias resoluções que regulamentam a obtenção, qualidade e distribuição (venda) do fitoterápico. O Ministério da Saúde (MS) disponibiliza a utilização de 12 medicamentos fitoterápicos na rede pública de saúde e estes medicamentos estão diretamente relacionados ao tratamento de doenças na atenção básica como: hipercolesterolemia leve a moderada, queimaduras, inflamação, infecções, obstipação intestinal eventual, broncoespasmo e broncoconstrição. Vários estudos comprovam os benefícios dos fitoterápicos, principalmente de forma complementar para promoção e recuperação da saúde da população.

Palavras-chave: Medicamentos fitoterápicos; Plantas medicinais; Atenção básica; Fitoterapia.

\begin{abstract}
Medicinal plants have been considered a solution to public health problems. The World Health Organization (WHO) emphasizes that up to $80 \%$ of the population of developing countries trust Complementary or Alternative Medicine (CAM) in initial health care. Thus, the research aims to describe the importance of using herbal medicines as an alternative and complementary treatment in primary care. The present study is an integrative literature review with descriptive content and qualitative nature. Data for this study were obtained from the following databases: Virtual Health Library (BVS), Scielo, Lilacs, medline and from the Academic Google directory. Original studies available and fully published that addressed the guiding question, between the years 2000 and 2020, were considered; in Portuguese, English or Spanish. Results: Aiming at greater safety and greater control in the use of herbal medicines, ANVISA (National Health Surveillance Agency) has several resolutions that regulate the procurement, quality and distribution (sale) of herbal medicines. The Ministry of Health (MS) provides the use of 12 herbal medicines in the public health network and these drugs are directly related to the treatment of diseases in primary care such as: mild to moderate hypercholesterolemia, burns, inflammation, infections, occasional constipation, bronchospasm and bronchoconstriction. Several studies prove the benefits of herbal medicines, especially in a complementary way for promoting and restoring the health of the population.
\end{abstract}

Keywords: Herbal medicines; Medicinal plants; Primary care; Herbal medicine. 


\begin{abstract}
Resumen
Las plantas medicinales se han considerado una solución a los problemas de salud pública. La Organización Mundial de la Salud (OMS) enfatiza que hasta el $80 \%$ de la población de los países en desarrollo confía en la Medicina Complementaria o Alternativa (MCA) en la atención médica inicial. Así, la investigación tiene como objetivo describir la importancia del uso de las hierbas medicinales como tratamiento alternativo y complementario en la atención primaria. El presente estudio es una revisión integradora de la literatura con contenido descriptivo y carácter cualitativo. Los datos para este estudio se obtuvieron de las siguientes bases de datos: Virtual Health Library (BVS), Scielo, Lilacs, medline y del directorio Academic Google. Se consideraron los estudios originales disponibles y completamente publicados que abordaron la pregunta orientadora, entre los años 2000 y 2020; en portugués, inglés o español. Resultados: Con el objetivo de una mayor seguridad y un mayor control en el uso de medicamentos a base de hierbas, ANVISA (Agencia Nacional de Vigilancia Sanitaria) tiene varias resoluciones que regulan la adquisición, calidad y distribución (venta) de medicamentos a base de hierbas. El Ministerio de Salud (MS) proporciona el uso de 12 medicamentos herbales en la red de salud pública y estos medicamentos están directamente relacionados con el tratamiento de enfermedades en atención primaria como: hipercolesterolemia leve a moderada, quemaduras, inflamación, infecciones, estreñimiento ocasional, broncoespasmo y broncoconstricción. Varios estudios demuestran los beneficios de las medicinas a base de hierbas, especialmente de forma complementaria para promover y restaurar la salud de la población.
\end{abstract}

Palabras clave: Hierbas medicinales; Plantas medicinales; Atención primaria; Fitoterapia.

\title{
1. Introdução
}

As plantas medicinais vem sendo uma solução considerada nos problemas de saúde pública. A Organização Mundial de Saúde (OMS) ressalta que até $80 \%$ da população de países em desenvolvimento confiam na Medicina Complementar ou Alternativa (MAC) em cuidados iniciais a saúde, devido os saberes tradicionais ou falta de alternativas (Baracho et al., 2006).

Segundo a OMS estima que o uso de algum tipo de medicina tradicional ou popular, presente nos cuidados básicos da saúde, e de aproximadamente $80 \%$ da população mundial, sendo as plantas medicinais correspondente a $85 \%$ desses tratamentos (Moraes et al., 2011).

A fitoterapia e o uso de plantas medicinais estão incluídos no conhecimento da medicina popular, onde constitui um conjunto de ideias enraizadas nos diversos usuários e praticantes dessa terapia, especialmente pela tradição oral (Bruning et al., 2012). O termo fitoterapia foi dado à terapêutica que utiliza os medicamentos cujos constituintes ativos são plantas ou derivados vegetais, que se originaram através do conhecimento do uso popular. As plantas utilizadas para esse fim são tradicionalmente consideradas medicinais (Brasil, 2012).

O uso de fitoterápicos com finalidade profilática, curativa, paliativa ou com fins de diagnóstico teve reconhecimento oficial pela OMS somente em 1978 através da a Declaração de Alma-Ata, quando recomendou mundialmente a necessidade dos conhecimentos para o seu uso. As plantas medicinais tornaram-se importantes instrumentos da Assistência Farmacêutica, com isso, a OMS publicou várias resoluções com o objetivo de expressar a posição do organismo a respeito da necessidade da valorização do uso desses medicamentos, no âmbito sanitário, principalmente na atenção básica (Brasil, 2006a).

Segundo estimativa do Ministério da Saúde (Brasil, 2012), aproximadamente 80\% da população brasileira utiliza produtos fitoterápicos. Conforme Reis (2018) o emprego da fitoterapia à Atenção Primária contribui para resgatar conhecimentos populares e culturais, contribuindo concomitantemente para aumentar o vínculo entre os pacientes e à equipe de saúde e permitindo que este seja um agente ativo em seu processo de saúde/adoecimento.

Os fitoterápicos proporcionam uma parte significativa no mercado de medicamentos. Por ano, esse setor movimenta US\$ 21,7 bilhões mundialmente. No Brasil, não existem dados oficiais atualizados, porém, enfatiza que esse mercado percorre cerca de US\$160 milhões por ano. Esse crescimento está vinculado principalmente a vendas internas, que obteve mais de 15\% anuais, comparada com a vendas dos medicamentos sintéticos que foi de aproximadamente $4 \%$. Considerando toda a cadeia produtiva, o setor fitoterápico movimenta cerca de $\mathrm{R} \$ 1$ bilhão anualmente (Carvalho et al.,2008). 
A OMS incentiva a utilização de plantas medicinais, ressaltando sua importância nos cuidados a saúde, recomendando a criação de programas mundialmente com objetivo de identificar e validar as plantas utilizadas na medicina tradicional, bem como garantir o controle, a qualidade e a segurança dos fitoterápicos (Oliveira et al., 2007).

Nesse contexto, com o objetivo de garantir a integralidade da atenção no âmbito do Sistema Único de Saúde (SUS), principalmente na atenção básica, foi promulgada em 2006, em portaria do Ministério da Saúde, a incorporação da medicina complementar alternativa (MCA) na rede pública de saúde no Brasil, com a finalidade de amparar e divulgar todos os conhecimentos tradicionais associados ao uso dos fitoterápicos (Boccolini \& Boccolini, 2020).

Grande foram os avanços com a elaboração e implementação de políticas públicas, programas e resoluções nos últimos anos, com o objetivo de valorizar as plantas medicinais e derivados nos cuidados primários com a saúde e sua inserção na rede pública. Atualmente, os principais instrumentos norteadores para o desenvolvimento das ações/programas com plantas medicinais e fitoterapia são: a Política Nacional de Práticas Integrativas e Complementares no SUS e a "Política Nacional de Plantas Medicinais e Fitoterápicos" (Brasil, 2012; Brasil, 2006b).

Portanto, o objetivo do estudo foi descrever a importância do uso de fitoterápicos como prática alternativa ou complementar na atenção básica.

\section{Metodologia}

\subsection{Desenho do estudo}

O presente estudo trata-se de uma revisão integrativa da literatura com teor descritivo e de natureza qualitativa, a qual teve como objetivo analisar a importância do uso de fitoterápicos como terapia alternativa e/ ou complementar utilizados na atenção básica (Botelho et al., 2011).

\subsection{Obtenção de dados}

Os dados para esse estudo foram obtidos através de artigos científicos, disponíveis nas bases de dados: Biblioteca Virtual em Saúde (BVS), Scientific Eletronic Libary Online (SciELO), Literatura Latino-Americana e do Caribe em Ciências da Saúde (LILICS), Medical Literature Analysis and Retrieval System Online (Medline) e no diretório Google Acadêmico. Utilizaram-se como descritores os termos: medicamentos fitoterápicos, plantas medicinais, atenção básica, fitoterapia. Inglês: herbal medicines, medicinal plants, primary care, herbal medicine.

Para elaboração dessa revisão foram seguidas as seguintes etapas: seleção da questão norteadora; busca dos artigos nas bases de dados utilizando-se os descritores e seus correspondentes em língua inglesa; seleção dos trabalhos encontrados mediante os critérios de inclusão e exclusão pré-determinados; avaliação dos estudos pré-selecionados; organização dos estudos selecionados; análise e interpretação dos resultados e apresentação da revisão (Botelho et al., 2011).

\subsection{Critérios de inclusão e exclusão}

A seleção dos artigos foi realizada com base nos critérios de inclusão: estudos originais disponíveis e publicados na íntegra que abordassem, no título ou resumo, respostas para a questão norteadora; que tivessem sido publicados entre os anos de 2000 e 2020; publicados nos idiomas português, inglês ou espanhol.

Foram excluídos trabalhos publicados em outras bases de dados, artigos disponíveis apenas após o pagamento. 
Figura 1 - fluxograma de estudos obtidos nas bases de dados.

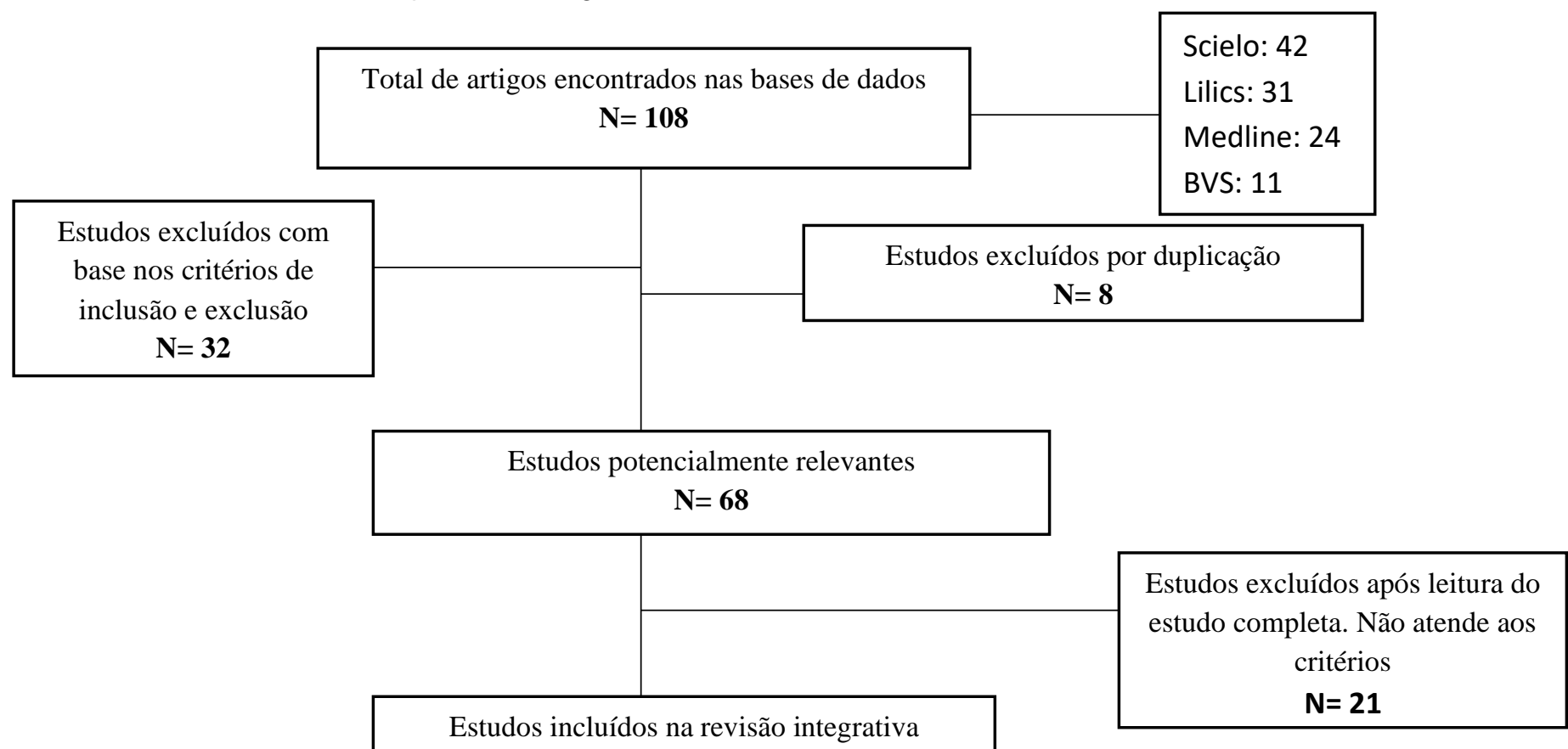

Fonte: Autores (2021).

\section{Resultados e Discussão}

\subsection{Legislação dos fitoterapicos}

Visando uma maior segurança e maior controle na utilização de medicamentos fitoterápicos, a ANVISA (Agência Nacional de Vigilância Sanitária), dispõe de várias resoluções (RDC: Resolução da Diretoria Colegiada) que regulam a obtenção, qualidade e distribuição (venda) do fitoterápico (Carvalho, 2007).

De acordo com RDC n8/04 da ANVISA, fitoterápico é definido como:

Medicamento obtido empregando-se exclusivamente matérias-primas ativas vegetais. É caracterizado pelo conhecimento da eficácia e dos riscos de seu uso, assim como pela reprodutibilidade e constância de sua qualidade. Sua eficácia e segurança é validada através de levantamentos etnofarmacológicos de utilização, documentações tecnocientíficas em publicações ou ensaios clínicos fase 3. Não se considera medicamento fitoterápico aquele que, na sua composição, inclua substâncias ativas isoladas, de qualquer origem, nem as associações destas com extratos vegetais (Brasil, 2004).

No Brasil, o registro de fitoterápicos baseia-se norma sanitária, a RDC nº 14/2010, "Esta Resolução possui o objetivo de estabelecer os requisitos mínimos para o registro de medicamentos fitoterápicos". Apresenta nível de exigências semelhante ao dos medicamentos sintéticos no que corresponde à qualidade dos produtos. Essa norma estabelece requisitos específicos para a validação do registro dos fitoterápicos e sua renovação, como Relatório Técnico, Relatório de Produção e Controle de Qualidade e Relatório de Eficácia e Segurança. Também dispõe sobre o registro de produtos importados e da renovação do registro, visando a garantia de qualidade nos produtos fitoterápicos produzidos.

Segundo A RDC n ${ }^{\circ}$ 14/2010 estabelece medidas de segurança e eficácia após sua comprovação.

Parágrafo único. Para os medicamentos fitoterápicos que comprovarem segurança e eficácia por tradicionalidade de uso, deve ser inserida a seguinte frase na bula, embalagem e material publicitário: "Medicamento registrado com base no uso tradicional, não sendo recomendado seu uso por período prolongado. Art. 19. Quando a comprovação da 
segurança e eficácia for efetuada por meio da "Lista de medicamentos fitoterápicos de registro simplificado", publicada pela ANVISA na IN 5, de 11 de dezembro de 2008, ou suas atualizações, o solicitante deve seguir integralmente as especificações ali definidas: parte usada, padronização/marcador, formas de uso, indicações/ações terapêuticas, dose diária, via de administração, concentração da forma farmacêutica, quando descrita, e restrição de uso. Parágrafo único. Poderão ser formuladas outras formas farmacêuticas, na mesma via de administração, desde que sejam apresentados os cálculos de equivalência de doses entre as formas extrativas e as formas farmacêuticas propostas (Brasil, 2010).

A RDC N²6, DE 13 DE MAIO DE 2014 "Dispõe sobre o registro de medicamentos fitoterápicos e o registro e a notificação de produtos tradicionais fitoterápicos."

Esta Resolução define as categorias de medicamento fitoterápico e produto tradicional fitoterápico e estabelece os requisitos mínimos para o registro e renovação de registro de medicamento fitoterápico, e para o registro, renovação de registro e notificação de produto tradicional fitoterápico. Estabelece que são considerados medicamentos fitoterápicos os obtidos com emprego exclusivo de matérias-primas ativas vegetais cuja segurança e eficácia sejam baseadas em evidências clínicas e que sejam caracterizados pela constância de sua qualidade. Também são considerados produtos tradicionais fitoterápicos os obtidos com emprego exclusivo de matérias-primas ativas vegetais cuja segurança e efetividade sejam baseadas em dados de uso seguro e efetivo publicados na literatura técnicocientífica e que sejam concebidos para serem utilizados sem a vigilância de um médico para fins de diagnóstico, de prescrição ou de monitorização (Brasil, 2010).

A seguir, apresenta-se a (Tabela 1) que fornece resoluções para o registro e regulamentação de fitoterápicos no Brasil, acompanhada da (Tabela 2) que ressalta outras resoluções para a regulamentação dos medicamentos fitoterápicos.

Tabela 1: Lista de resoluções para o registro e regulamentação dos fitoterápicos no brasil.

\begin{tabular}{l|l}
\hline Resolução 196/96 e 251/97(CNS) & $\begin{array}{l}\text { Estabelece Diretrizes e Normas Regulamentadoras de Pesquisas } \\
\text { envolvendo Seres Humanos, relacionada a segurança e eficácia do } \\
\text { uso de medicamentos. }\end{array}$ \\
\hline ; RDC 48 de 16/03/2004 (ANVISA) & $\begin{array}{l}\text { aprova o Regulamento Técnico, visando a atualização a } \\
\text { normatização do registro de medicamentos fitoterápicos, onde são } \\
\text { estabelecidos todos os requisitos necessários para a sua concessão. }\end{array}$ \\
\hline Resolução - RE n ${ }^{\circ}$ 88/04 (ANVISA) & $\begin{array}{l}\text { Lista as referências bibliográficas para avaliação de segurança e } \\
\text { eficácia de fitoterápicos. }\end{array}$ \\
\hline Resolução - RE n ${ }^{\circ} 89$ (ANVISA) & estabelece a “Lista de Registro Simplificado de Fitoterápicos” \\
\hline Resolução - RE n ${ }^{\circ} 90 / 04$ (ANVISA) & $\begin{array}{l}\text { estabelece a publicação da "Guia para a Realização de Estudos de } \\
\text { Toxicidade Pré-Clínica de Fitoterápicos }\end{array}$ \\
\hline Resolução - RE no 91/04 & $\begin{array}{l}\text { Determina a publicação da guia para realização de alterações, } \\
\text { inclusões, notificações e cancelamentos pós-registro. }\end{array}$ \\
\hline
\end{tabular}

Fonte: Autores.

Tabela 2: Outras resoluções sobre o regulamento dos fitoterápicos.

\begin{tabular}{l|l}
\hline Portaria ${ }^{\circ} 110 / 97$ e RDC $n^{\circ} 140 / 03$ & Estabelece informações de bula. \\
\hline${\operatorname{RDC~} n^{\circ} 333 / 03}^{R_{C} n^{\circ} 138 / 03 \text { e RDC n }{ }^{\circ} 98 / 16}$ & Normas de rotulagem de medicamentos \\
\hline $\operatorname{RE~}^{\circ} 01 / 05$ & enquadramento na categoria de venda de medicamentos. \\
\hline RDC $n^{\circ} 899 / 03$ & Guia para a Realização de Estudos de Estabilidade \\
\hline IN 5/08 & $\begin{array}{l}\text { Guia para Realização de Validação de Metodologia } \\
\text { Analítica. }\end{array}$ \\
\hline
\end{tabular}

Fonte: Adaptada de Carvalho (2007).

\subsection{A importâcia da fitoterapia}

A palavra fitoterapia é derivada do grego "therapeia" que significa tratamento e "phyton": vegetal. É uma ciência que estuda as plantas para fins medicinais e para obtenção de cura em diversas doenças, com diferentes formas de apresentação 
farmacêutica. Essa pratica promove e favorece o desenvolvimento cientifico e cultural, com a participação social na prevenção de agravos e promoção à saúde. Reforça uma escolha mais natural e menos nocivo à saúde, principalmente se comparada ao uso em excesso de medicamentos alopáticos (Melo et al., 2019).

O Brasil, é um dos países que pode contribuir significativamente para o desenvolvimento da fitoterapia, pois detém de uma grande biodiversidade e solo rico em quase todo seu território, conta com uma imensa gama de plantas medicinais, com várias espécies já identificadas, facilitando o acesso da população a uma medicina mais barata (Brandão et al., 2006). A OMS incentiva a sociedade brasileira ao uso de produtos naturais, especialmente a população de baixa renda e que tenha acesso mais restrito aos medicamentos sintéticos, a fitoterapia para essa comunidade e uma alternativa essencial pelo seu baixo custo. Ademais, está terapia possui certas vantagens em relação a alopatia, por exemplo, efeitos colaterais menores quando corretamente usada (Santos et al., 2013).

Segundo Queiroz (2000) a revitalização das práticas médicas antigas, hoje considerada medicina integrativa, é um fenômeno que contribui para a forma hegemônica gradual destas modalidades, uma vez que sua organização mais ampla e integrada permite respostas mais apropriadas aos problemas gerados pela mecanicista especialização excessiva dos métodos médicos convencionais.

A fitoterapia é a pratica integrativa medicinal que obteve um crescimento mais significativo durante esses anos. A comercialização de fitofármacos mundialmente percorre em torno de 15 bilhões de dólares. O principal fator responsável pelo crescimento está presente na evolução dos estudos científicos, ligado diretamente a descoberta da eficácia de plantas medicinais, principalmente as utilizadas pela população para fins medicinais, através dos estudos químicos e farmacológicos (Santos et al., 2011).

Sendo assim, Fontenele et al. (2013) promoveu um levantamento dos principais motivos para a implantação de projetos de fitoterapia apontados pelos estados/municípios para o Ministério da Saúde conforme o (Quadro 1).

Quadro 1: Principais motivos de implantação da fitoterapia na atenção primária.

\begin{tabular}{|c|}
\hline a ampliação do acesso da população aos medicamentos \\
\hline grande aceitação da população/resgate da cultura popular/solicitação da comunidade \\
\hline baixo custo \\
\hline orientar à população quanto ao uso correto das plantas medicinais \\
\hline baixo número de efeitos colaterais e eficácia comprovada \\
\hline Fonte: Elaborada pelos autores.
\end{tabular}

Contudo, é essencial que o uso da fitoterapia na atenção básica seja avaliado e planejado em conjunto com os diversos fatores que permeiam a atenção à saúde, inclusive a aceitação e o reconhecimento, pelos profissionais envolvidos, das práticas terapêuticas (Fontenele et al., 2013).

\subsection{Fitoterapia no SUS}

Na década de 70 a Organização Mundial de Saúde (OMS), criou o "Programa de Medicina Tradicional", incentivando o estabelecimento de políticas públicas que refletisse sobre a Medicina Tradicional e a Medicina Complementar e Alternativa (Batista \& Valença, 2012).

Nesse entendimento, em 1986 foi realizada a $8^{\text {a }}$ Conferência Nacional de Saúde que teve como objetivo a elaboração das práticas alternativas de assistência à saúde no âmbito dos serviços de saúde. Posteriormente, com a $10^{\mathrm{a}}$ Conferência Nacional de Saúde, em setembro de 1996, em Brasília, que teve como proposta integrar ao SUS as terapias alternativas e práticas populares, que promoveu em conjunto com profissionais de saúde e especialistas, o emprego de normas para a utilização da fitoterapia e da homeopatia na assistência da saúde pública (Andrade et al., 2017; Brasil, 2006a). 
Em 2006, através do Decreto da Presidência da República nº. 5.813, de 22 de junho, foi criada a Política Nacional de Plantas Medicinais e Fitoterápicos (PNPMF) com objetivo de efetivar o melhor conhecimento sobre os fitoterápicos na saúde pública, visando promover uma melhor qualidade de vida a população brasileira (Almeida, 2011; Brasil, 2006b). No mesmo ano, através da portaria do Ministério da Saúde GM/MS nº 971, já havia sido criada a Política Nacional de Práticas Integrativas e Complementares no SUS (PNPIC), envolvendo, além da Fitoterapia, a Homeopatia, a Medicina Tradicional Chinesa/Acupuntura, o Termalismo/Crenoterapia e a Medicina Antroposófica. A implantação da PNPIC teve o objetivo oferecer à população brasileira esses serviços e produtos relacionados à fitoterapia, acupuntura, homeopatia e termalismo social no âmbito do SUS, assegurando a Integralidade na atenção à saúde (Ibiapina et al.,2014). Essas políticas promoveram o debate sobre os mecanismos de implementação da Fitoterapia nos serviços de saúde da atenção básica, e diferentes opiniões sobre como deveria ocorrer (Figueredo et al.,2014).

Posteriormente à aprovação destas políticas, através de suas diretrizes, instituiu-se um Grupo de Trabalho Interministerial (GTI) para elaborar o Programa Nacional de Plantas Medicinais e Fitoterápicos pela Portaria Interministerial $\mathrm{n}^{\circ}$ 2.960/2008. Esta Portaria também criou o Comitê Nacional de Plantas Medicinais e Fitoterápicos, com a função de monitorar e avaliar a implantação da PNPIC. Essas políticas buscam incorporar e implementar tais práticas no âmbito do SUS, com a concepção da recuperação, prevenção e promoção a saúde, na Atenção Básica, por essa ser a porta de entrada no sistema se saúde. Ademais, têm a finalidade de aumentar o acesso a tratamento opcionais com produtos seguros, eficazes e de qualidade, sem o propósito de substituir o modelo convencional (Batista \&Valença, 2012; Brasil, 2011; Brasil, 2016).

\subsection{Os fitoterápicos presente no SUS e suas vantagens}

A elaboração de políticas nacionais para o uso das plantas medicinais e dos fitoterápicos e resulta de batalhas anteriores à criação do SUS, em que diversos atores, como pesquisadores, gestores, profissionais de saúde e usuários tiveram papel fundamental (Brasil, 2006a).

Para Figueredo et al. (2014) A implementação da Fitoterapia no SUS representa, além da incorporação de mais uma terapêutica ao arsenal de possibilidades de tratamento à disposição dos profissionais de saúde, o resgate de uma prática milenar, onde se imbricam o conhecimento científico e o conhecimento popular e seus diferentes entendimentos sobre o adoecimento e as formas de tratá-lo.

No Brasil, a Farmacopeia Brasileira e quem estabelece sobre informações científicas sobre segurança, eficácia e controle de qualidade das plantas medicinais e fitoterápicos, acrescida do Memento Fitoterápico, que é um conjunto de monografias com informações referentes ao uso terapêutico das plantas medicinais, e também pelo Formulário de Fitoterápicos, onde estão contidas as informações sobre a forma correta de preparo de formulações oficiais e as indicações e restrições de uso de cada espécie de planta medicinal. Esses documentos foram elaborados pela ANVISA e auxiliam na pratica de manipulação e dispensação de fitoterápicos nos programas de Fitoterapia do SUS (Dias et al.,2017).

Desde 2007, o Brasil através do sistema público de saúde oferece fitoterápicos derivados de plantas. Atualmente, o Ministério da Saúde (MS) disponibiliza a utilização de 12 medicamentos fitoterápicos na rede pública de saúde através da RENAME conforme a (Tabela 3) (Marmitt et al., 2015).

A Relação nacional de Medicamentos Essenciais (RENAME), é uma lista de fármacos com o intuito de atender as necessidades básicas da população. Esse documento rege o atendimento farmacológico no âmbito do SUS, sendo considerado como uma ferramenta imprescindível para a promoção do acesso e uso racional de medicamentos (Brasil, 2015). 
Tabela 3: Relação nacional de Medicamentos Essenciais (RENAME)

\begin{tabular}{|c|c|c|}
\hline Nome popular & Nome científico & indicação \\
\hline Alcachofra & Cynara scolymus $L$. & $\begin{array}{l}\text { Tratamento dos sintomas de dispepsia funcional } \\
\text { (síndrome do desconforto } \\
\text { pós-prandial) e de hipercolesterolemia leve a } \\
\text { moderada. Apresenta ação colagoga e colerética }\end{array}$ \\
\hline Aroeira & (Schinus terebinthifolius Raddi & $\begin{array}{l}\text { Apresenta ação cicatrizante, anti-inflamatória e } \\
\text { anti-séptica tópica, para uso ginecológico }\end{array}$ \\
\hline Babosa & Aloe vera (L.) Burm.f & $\begin{array}{l}\text { Tratamento tópico de queimaduras de } 1^{\circ} \text { e } 2^{\circ} \\
\text { graus como coadjuvante } \\
\text { nos casos de Psoríase vulgaris }\end{array}$ \\
\hline Cáscara-sagrada & Rhamnus purshiana D.C & $\begin{array}{l}\text { Auxilia nos casos de obstipação intestinal } \\
\text { eventual }\end{array}$ \\
\hline Espinheira-santa & Maytenus officinalis Mabb & $\begin{array}{l}\text { Auxilia no tratamento de gastrite e úlcera } \\
\text { duodenal e sintomas de dispepsias }\end{array}$ \\
\hline Guaco & Mikania glomerata Spreng & $\begin{array}{l}\text { Apresenta ação expectorante e broncodilatadora. } \\
\text { Atua relaxando musculatura lisa das vias aéreas } \\
\text { superiores. }\end{array}$ \\
\hline Garra-do-diabo & Harpagophytum procumbens & $\begin{array}{l}\text { Tratamento da dor lombar baixa aguda e como } \\
\text { coadjuvante nos casos de osteoartrite. Apresenta } \\
\text { ação anti-inflamatória }\end{array}$ \\
\hline Hortelã & Mentha $x$ piperita $L$ & $\begin{array}{l}\text { Tratamento da síndrome do cólon irritável. } \\
\text { Apresenta ação antiflatulenta e } \\
\text { Antiespasmódica }\end{array}$ \\
\hline Isoflavona de soja & Glycine $\max ($ L.) Merr & Auxilia no alívio dos sintomas do climatério \\
\hline Plantago & Plantago ovata Forssk & $\begin{array}{l}\text { Auxilia nos casos de obstipação intestinal } \\
\text { habitual. Tratamento da síndrome do cólon } \\
\text { irritável }\end{array}$ \\
\hline Salgueiro & Salix alba $L$ & $\begin{array}{l}\text { Tratamento de dor lombar baixa aguda. } \\
\text { Apresenta ação antiinflamatória }\end{array}$ \\
\hline Unha-de-gato & Uncaria tomentosa & $\begin{array}{l}\text { Auxilia nos casos de artrites e osteoartrite. } \\
\text { Apresenta ação antiinflamatória e } \\
\text { imunomoduladora }\end{array}$ \\
\hline
\end{tabular}

Fonte: Elaboradas pelos autores.

No ano de 2009, o Ministério da Saúde divulgou a Relação Nacional de Plantas Medicinais de Interesse ao Sistema Único de Saúde (RENISUS). Onde consta várias plantas medicinais que apresentam potencial terapêutico para gerar produtos de interesse ao SUS. As espécies contidas nessa lista, provem da sabedoria popular e cientificamente comprovadas. A elaboração dessa lista é um passo importante, pois direciona a pesquisa clínica e o estudo para este conjunto de plantas (Marmitt et al., 2015). 
Nesse sentido, o governo implementou a RENISUS uma lista com 71 plantas medicinais utilizadas para fins medicinais nas diversas regiões do Brasil (Quadro 2), tendo em vista a produção de medicamentos fitoterápicos no âmbito do SUS, observando tanto o tratamento, como também a prevenção de doenças. A RENISUS traz apenas o nome científico das espécies vegetais, porque ainda estão sendo levantadas informações sobre essas espécies (Silva, 2019; Brasil, 2009).

Quadro 2 - RENISUS - Relação Nacional de Plantas Medicinais de Interesse ao SUS Espécies vegetais.

\begin{tabular}{|c|c|}
\hline 1 & Achillea millefolium \\
\hline 2 & Allium sativum \\
\hline 3 & Aloe spp* (A. vera ou A. barbadensis) \\
\hline 4 & Alpinia spp* (A. zerumbet ou A. speciosa) \\
\hline 5 & Anacardium occidentale \\
\hline 6 & Ananas comosus \\
\hline 7 & Apuleia ferrea $=$ Caesalpinia ferrea $*$ \\
\hline 8 & Arrabidaea chica \\
\hline 9 & Artemisia absinthium \\
\hline 10 & Baccharis trimera \\
\hline 11 & $\begin{array}{l}\text { Bauhinia } \operatorname{spp}^{*} \text { (B. affinis, B. forficata ou } B . \\
\text { variegata) }\end{array}$ \\
\hline 12 & Bidens pilosa \\
\hline 13 & Calendula officinalis \\
\hline 14 & Carapa guianensis \\
\hline 15 & Casearia sylvestris \\
\hline 16 & $\begin{array}{l}\text { Chamomilla recutita }=\text { Matricaria } \\
\text { chamomilla }=\text { Matricaria recutita }\end{array}$ \\
\hline 17 & Chenopodium ambrosioides \\
\hline 18 & Copaifera spp* \\
\hline 19 & 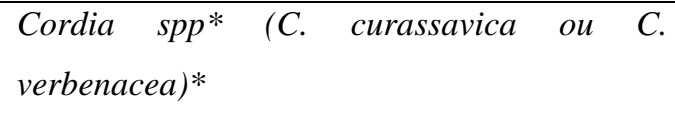 \\
\hline 20 & Costus spp* (C. scaber ou C. spicatus) \\
\hline 21 & Croton spp (C. cajucara ou C. zehntneri) \\
\hline 22 & Curcuma longa \\
\hline 23 & Cynara scolymus \\
\hline 24 & Dalbergia subcymosa \\
\hline 25 & Eleutherine plicata \\
\hline 26 & Equisetum arvense \\
\hline 27 & Erythrina mulungu \\
\hline 28 & Eucalyptus globulus \\
\hline 29 & Eugenia uniflora ou Myrtus brasiliana* \\
\hline
\end{tabular}

\begin{tabular}{|c|c|}
\hline 37 & Lippia sidoides \\
\hline 38 & Malva sylvestris \\
\hline 39 & Maytenus spp* (M. aquifolium ои M. ilicifolia) \\
\hline 40 & Mentha pulegium \\
\hline 41 & Mentha spp* (M. crispa, M. piperita ou M. villosa) \\
\hline 42 & Mikania spp* (M. glomerata ou M. laevigata) \\
\hline 43 & Momordica charantia \\
\hline 44 & Morus sp* \\
\hline 45 & Ocimum gratissimum \\
\hline 46 & Orbignya speciosa \\
\hline 47 & Passiflora spp* (P. alata, $P$. edulis ou $P$. incarnata $)$ \\
\hline 48 & Persea $\operatorname{spp}^{*}(P$. gratissima ou $P$. americana $)$ \\
\hline 49 & Petroselinum sativum \\
\hline 50 & $\begin{array}{l}\text { Phyllanthus spp* }{ }^{*} \text { P. amarus, P.niruri, P. tenellus e } \\
\text { P. urinaria) }\end{array}$ \\
\hline 51 & Plantago major \\
\hline 52 & Plectranthus barbatus $=$ Coleus barbatus \\
\hline 53 & Polygonum spp* (P. acre ou P. hydropiperoides) \\
\hline 54 & Portulaca pilosa \\
\hline 55 & Psidium guajava \\
\hline 56 & Punica granatum \\
\hline 57 & Rhamnus purshiana \\
\hline 58 & Ruta graveolens \\
\hline 59 & Salix alba \\
\hline 60 & Schinus terebinthifolius $=$ Schinus aroeira \\
\hline 61 & Solanum paniculatum \\
\hline 62 & Solidago microglossa \\
\hline 63 & $\begin{array}{l}\text { Stryphnodendron adstringens }=\text { Stryphnodendron } \\
\text { barbatimam }\end{array}$ \\
\hline 64 & Syzygium spp* (S. jambolanum ou $S$. cumini) \\
\hline 65 & Tabebuia avellanedeae \\
\hline
\end{tabular}


Research, Society and Development, v. 11, n. 1, e44611124643, 2022

(CC BY 4.0) | ISSN 2525-3409 | DOI: http://dx.doi.org/10.33448/rsd-v11i1.24643

\begin{tabular}{|l|l|}
\hline 30 & Foeniculum vulgare \\
\hline 31 & Glycine max \\
\hline 32 & Harpagophytum procumbens \\
\hline 33 & Jatropha gossypiifolia \\
\hline 34 & Justicia pectoralis \\
\hline 35 & Kalanchoe pinnata $=$ Bryophyllum calycinum \\
\hline 36 & Lamium album \\
\hline
\end{tabular}

\begin{tabular}{|l|l|}
\hline 66 & Tagetes minuta \\
\hline 67 & Trifolium pratense \\
\hline 68 & Uncaria tomentosa \\
\hline 69 & Vernonia condensata \\
\hline 70 & Vernonia spp* V. ruficoma ou V. polyanthes) \\
\hline 71 & Zingiber officinale \\
\hline
\end{tabular}

Fonte: DAF/SCTIE/MS - RENISUS - 2009.

A classificação das plantas medicinais pode ser realizada por categorias, conforme sua ação terapêutica: estimulantes, calmantes, emolientes, fortificantes, de ação coagulante, diuréticas, sudoríferas, hipotensoras, de função reguladora intestinal, colagogas, depurativas, remineralizantes e reconstituintes. Algumas vantagens das plantas medicinais são a sua eficácia, seu baixo risco prejudicial à saúde, além da sua qualidade e segurança. Sendo assim, as etapas de produção dos fitoterápicos devem ser elaboradas e analisados corretamente tais como sua manipulação, formulação e dispensação através do trabalho multidisciplinar, para melhor qualidade de vida da população. (Armous, Santos \& Beinner, 2005).

Os medicamentos fitoterápicos devem possuir critérios para sua aprovação como: a garantia de qualidade, efeitos terapêuticos comprovados, e uma composição padronizada e estável. A eficácia e a segurança destes produtos podem ser autenticadas por meio de levantamentos etnofarmacológicos, documentações tecnocientíficas, estudos farmacológicos e toxicológicos pré-clínicos e clínicos das plantas medicinais (Zardeto-sabec et al., 2019).

segundo Pinha et al. (2019) acredita que o saber da população ainda é fundamental para o descobrimento de novas espécie e ações dessas plantas, por sua vez a população utiliza e resulta em melhoria de vida, adquirindo experiência e explorando plantas inacessíveis de muitos locais. Uma vez a planta apresentando ações benéficas, podendo resultar em um campo de estudo instigante e relevante para a ciência. Afins de inverter o quadro de declínio da prática, uso e perda de conhecimento de geração a geração, causado pela facilidade de acesso e concorrência com produtos modernos fármacos.

\subsection{Principais doenças na atençao primária e seu tratamento por fitoterápicos}

A fitoterapia tem comprovado ser um pratica terapêutica bastante eficiente, pois fornece eficácia, segurança e o baixo custo operacional na sua utilização, ligados a programas de atenção básica à saúde, podendo diminuir a demanda de medicamentos nos serviços de saúde. Essa terapia tem mais relevância devido a facilidade de ser adquirida, pois o conhecimento popular, formulações caseiras e seu fácil preparo, proporcionam um tratamento mais acessível, principalmente em regiões onde culturalmente é comum o uso de plantas medicinas (Ibiapina et al., 2014).

A OMS incentiva fortemente o uso de plantas medicinais e fitoterápicos no tratamento de diversas doenças, visto que apresentam diversas aplicações terapêuticas e podem ser eficazes para muitas patologias comuns (Quadro 3), como feridas, constipação, dispepsia, artrite, hiperlipidemia, infecções do trato respiratório, dentre muitas outras (Lombardo, 2021). 
Quadro 3 - Principais doenças presentes na atenção básica e as referências científicas no tratamento com fitoterápicos.

\begin{tabular}{|c|c|c|c|}
\hline $\begin{array}{l}\text { AUTOR/ } \\
\text { ANO }\end{array}$ & TITULO & INDICAÇÃO & BENEFÍCIOS NO TRATAMENTO COM FITOTERÁPICOS. \\
\hline $\begin{array}{l}\text { Santos et al. } \\
(2018)\end{array}$ & $\begin{array}{l}\text { principais } \text { plantas } \\
\text { medicinais e fitoterapia na } \\
\text { terapêutica de afecções } \\
\text { estomacais: uma revisão } \\
\text { bibliográfica }\end{array}$ & $\begin{array}{l}\text { DOENÇAS } \\
\text { GASTROINTEST } \\
\text { INAIS }\end{array}$ & $\begin{array}{l}\text { As plantas medicinais promovem ação Antiácidas e } \\
\text { antiespasmódicas, ajuda o sistema digestório e biliar, além de } \\
\text { reduzi ácido gástrico promovendo a diminuição do muco, } \\
\text { amentando a concentração das prostaglandinas e produção de } \\
\text { novas células estomacais. }\end{array}$ \\
\hline $\begin{array}{l}\text { Vieira \& } \\
\text { Medeiros, } \\
(2019) .\end{array}$ & $\begin{array}{l}\text { a utilização de fitoterápicos } \\
\text { no tratamento da obesidade }\end{array}$ & OBESIDADE & $\begin{array}{l}\text { Diferentes plantas medicinais têm sido analisadas e utilizadas } \\
\text { com o objetivo de diminuição de peso, especialmente aquelas } \\
\text { com ação inibidora de lipases, termogênica, ou que reduzem o } \\
\text { apetite }\end{array}$ \\
\hline $\begin{array}{l}\text { Moreira et al. } \\
(2020) \text {. }\end{array}$ & $\begin{array}{l}\text { uso de medicamentos } \\
\text { fitoterápicos como opção } \\
\text { anti-inflamatória na } \\
\text { odontologia. }\end{array}$ & INFLAMAÇÃO & $\begin{array}{l}\text { afirmaram que os flavonoides e ácido cafeico presentes na } \\
\text { Própolis desempenham o papel na redução da resposta } \\
\text { inflamatória, inibindo ácido araquidônico e a síntese de } \\
\text { prostaglandina, além disso, ativam o timo e auxiliam o sistema } \\
\text { imunitário através da atividade fagocítica, outro mecanismo de } \\
\text { ação na diminuição da produção do nitrito, possuindo uma } \\
\text { resposta anti-inflamatória. }\end{array}$ \\
\hline $\begin{array}{l}\text { Lucena \& } \quad \text { Guedes, } \\
\text { (2020). }\end{array}$ & $\begin{array}{l}\text { o uso de fitoterápicos na } \\
\text { prevenção e no tratamento } \\
\text { da hipertensão arterial } \\
\text { sistêmica }\end{array}$ & HIPERTENSÃO & $\begin{array}{l}\text { Certas espécies de plantas podem diminuir os graus de } \\
\text { colesterol sanguíneo, preservar as células endoteliais vasculares } \\
\text { contra danos. Além disso, possuem a ação antitrombótica por } \\
\text { causa da elevação da atividade fibrinolítica e do restringimento } \\
\text { da associação plaquetária estimulando a diminuição da pressão } \\
\text { arterial correspondente ao efeito de relaxamento das células lisas, } \\
\text { ocasionando vasodilatação. }\end{array}$ \\
\hline $\begin{array}{l}\text { Pinto et al. } \\
(2020)\end{array}$ & $\begin{array}{l}\text { a fitoterapia no tratamento } \\
\text { de pele: um estudo } \\
\text { bibliográfico }\end{array}$ & $\begin{array}{l}\text { DOENÇAS DE } \\
\text { PELE }\end{array}$ & $\begin{array}{l}\text { afirmam a importância das plantas como uma nova tentativa da } \\
\text { medicina no combate às várias doenças e que os resultados têm } \\
\text { sido promissores, principalmente na cicatrização de feridas. } \\
\text { Muitos estudos têm concentrado esforços no desenvolvimento de } \\
\text { cosméticos, usando como base plantas medicinais, devido aos } \\
\text { efeitos nocivos causados por muitos produtos químicos. Esses } \\
\text { produtos têm sido usados no tratamento das feridas ao longo dos } \\
\text { anos, por promoverem a coagulação do sangue, combater } \\
\text { infecções e aceleram o processo de cicatrização. }\end{array}$ \\
\hline $\begin{array}{l}\text { Alvarenga et } \\
\text { al. (2017). }\end{array}$ & $\begin{array}{l}\text { uso de plantas medicinais } \\
\text { para o tratamento do } \\
\text { diabetes mellitus no vale do } \\
\text { paraíba-sp }\end{array}$ & DIABETES & $\begin{array}{l}\text { As plantas com efeito hipoglicêmico podem abaixar os níveis } \\
\text { séricos de glicose através de vários mecanismos, a saber: } \\
\text { aumento da liberação de insulina por estímulo às células } \beta \text { - } \\
\text { pancreáticas; resistência a hormônios responsáveis por elevar a } \\
\text { taxa de glicose; aumento da sensibilidade e do número de sítios } \\
\text { receptores a insulina; diminuição da degradação de glicogênio; } \\
\text { eliminação de radicais livres. }\end{array}$ \\
\hline $\begin{array}{l}\text { Reinecken } \\
\text { al. (2018). }\end{array}$ & $\begin{array}{l}\text { plantas medicinais } \\
\text { potencialmente }\end{array}$ & $\begin{array}{l}\text { HIPERLIPIDEMI } \\
\text { A }\end{array}$ & $\begin{array}{l}\text { obteve-se uma diminuição no índice aterogênico e um aumento } \\
\text { da relação do LDL/HDL, isso é, havendo uma diminuição dos }\end{array}$ \\
\hline
\end{tabular}




\begin{tabular}{|c|c|c|c|}
\hline & hipolipidêmicas & & $\begin{array}{l}\text { níveis do LDL e um aumento nos níveis do HDL. A planta } \\
\text { também apresentou efeitos antioxidantes, dado que a mesma } \\
\text { apresentou atividade de eliminação de radicais livres. O estudo } \\
\text { destaca que estes benefícios podem ter ocorrido devido a } \\
\text { presença de flavonoides na planta, que possuem atividades } \\
\text { antidislipidémicas e antioxidantes. }\end{array}$ \\
\hline $\begin{array}{l}\text { Darroz et al. } \\
\text { (2014). }\end{array}$ & $\begin{array}{l}\text { utilização de fitoterápicos } \\
\text { no tratamento de } \\
\text { constipação intestinal }\end{array}$ & $\begin{array}{l}\text { CONSTIPAÇÃO } \\
\text { INTESTINAL }\end{array}$ & $\begin{array}{l}\text { os laxantes utilizados clinicamente estão divididos } \\
\text { farmacologicamente em cinco tipos, no entanto somente em duas } \\
\text { dessas classes se encontram representantes fitoterápicos. } \\
\text { Primeiramente, têm-se os laxativos formadores de massa ou } \\
\text { incrementadores do bolo fecal, ou seja, aqueles que aumentam a } \\
\text { massa intestinal, cujo mecanismo de ação decorre de seu efeito } \\
\text { hidrofílico, com acúmulo de líquido no lúmen intestinal, aumento } \\
\text { de volume e amolecimento do bolo fecal. Na segunda classe têm- } \\
\text { se os laxativos secretagogos ou agentes com efeitos diretos nas } \\
\text { células epiteliais nervosas e da musculatura lisa ou agentes } \\
\text { irritantes, que estimulam diretamente o plexo mioentérico } \\
\text { aumentando a motilidade intestinal }\end{array}$ \\
\hline $\begin{array}{l}\text { Haeffner et al. } \\
\text { (2012). }\end{array}$ & $\begin{array}{l}\text { plantas medicinais } \\
\text { utilizadas para o alívio da } \\
\text { dor pelos agricultores } \\
\text { ecológicos do sul do brasil }\end{array}$ & DOR (GERAL) & $\begin{array}{l}\text { As plantas medicinais possuem diversos princípios ativos, entre } \\
\text { estes pode-se encontrar alguns alcalóides, mucilagens, } \\
\text { flavonóides, taninos e óleos essenciais, entre outras substâncias, } \\
\text { que atuam no mecanismo da analgesia. Estudos que abrangem } \\
\text { desde a etnobotânica até a farmacognosia, revelam efeitos sobre } \\
\text { os seres vivos em geral, como o analgésico, ampliando as formas } \\
\text { de terapêutica para diversas doenças. }\end{array}$ \\
\hline
\end{tabular}

Fonte: Elaboradas pelos autores.

Segundo Gadelha et al. (2015) afirma que várias espécies vegetais possuem propriedades terapêuticas, as mesmas representam a principal matéria médica utilizada pelas chamadas medicinas tradicionais. o uso de plantas medicinais representa um fator importante para a manutenção das condições de saúde das pessoas.

Porém, uso de plantas com fins terapêuticos, sem orientação adequada, e extremamente preocupante que deve ser considerado pelos atores sociais do setor de saúde, principalmente pelos educadores em saúde, informando a contra- indicação e a toxidade das espécies em caso de uso. Portanto, da mesma forma que as plantas são remédios poderosos e eficazes, existe o risco de intoxicação causada pelo seu uso indevido, e que deve ser de total importância. A observância das dosagens prescritas e o cuidado na identificação do produto utilizado podem evitar uma série de acidentes relacionados a fitoterapia (Tomazzoni et al., 2006).

\section{Considerações Finais}

As plantas medicinais representam fator de grande importância para a manutenção das condições de saúde das pessoas. Além da comprovação da ação terapêutica de várias plantas utilizadas popularmente, a fitoterapia representa parte importante da cultura de um povo, sendo também parte de um saber utilizado e difundido pelas populações ao longo de várias gerações. 
A presente pesquisa tem como objetivo contribuir para o conhecimento sobre o uso de fitoterápico na atenção básica como forma de tratamento alternativo. Vários estudos comprovam os benefícios dos fitoterápicos, principalmente de forma complementar para promoção e recuperação da saúde da população.

As resoluções presentes no Brasil sobre os fitoterápicos, tem como finalidade esclarecer e regulamentar plantas que através do conhecimento popular e comprovação cientifica tornaram-se essências no tratamento de diversas doenças, principalmente na atenção básica.

Portanto, e de total importância que o governo intensifique o uso da fitoterapia na sociedade brasileira, ademais, as publicações de estudos para maior descoberta de plantas medicinais que estão presente no cotidiano da população, com o intuito de promover e ampliar o número de medicamentos fitoterápicos presentes no SUS, visando sempre a promoção em saúde pública.

Por fim, recomenda-se para trabalhos futuros estudos sobre a atualização dos fitoterápicos, onde possa mostra a evolução da fitoterapia no Brasil. Ademais, a inclusão dessa pratica terapêutica no tratamento de doenças crônicas e sua comparação com tratamentos por medicamentos alopáticos.

\section{Referências}

Almeida, M. Z. (2011). Fitoterapia no SUS no Estado da Bahia: contribuição para valorização do conhecimento e das práticas tradicionais na rede básica de saúde. Revista Fitos, 6(1), 29-34.

Alvarenga, C. F., Lima, K. M. N., Mollica, L. R., Azeredo, L. O., \& Carvalho, C. (2017). Uso de plantas medicinais para o tratamento do diabetes mellitus no vale do paraíba-SP. Revista Ciências em Saúde, 2(2):36-44.

Arnous, A. H., Santos, A. S., \& Beinner, R. P. C. (2015). Plantas medicinais de uso caseiro conhecimento popular e interesse por cultivo comunitário. Revista Espaço para a Saúde, 6(2), 1-6.

Andrade, S. A. L., Tristão, M. I. S., Miguel, M. D., Dias, J. F. G.,Gomes, E. C., Burci, L. M., \& Paula, C. S. (2017). Fitoterápicos da relação nacional de medicamentos essenciais no Brasil. Revista Cubana de Plantas Medicinales, 22(1), 1-8.

Baracho, N. C. V., Silva, L. U. M. A., Alves, L. J., Braga L. T. P., Carneiro M. F. S., \& Siqueira, M. T. G. (2006). The use of medicinal plants as alternative treatment in the jardim das colinas district, itajubá, mg, brazil. Revista Médica de Minas Gerais,16(2), 88-91.

Batista, L. M., \& Valença, A. M. G. (2012). A Fitoterapia no Âmbito da Atenção Básica no SUS: Realidades e Perspectivas. Pesquisa Brasileira em Odontopediatria e Clínica Integrada,12(2), 96-293.

Boccolini, P. M. M., \& Boccolini, C. S. (2020). Prevalence of complementary and alternative medicine (CAM) use in Brazil. BMC Complementary Medicine and Therapies, 20(51), 1-10.

Botelho, L. L. R., Cunha, C. C. A., \&· Macedo, M. (2011). O método da revisão integrativa nos estudos organizacionais. Gestão e Sociedade, 5(11), $121-136$.

Brandão, M. G. L., Acurcio, F. A., Montemor, R. L. M., \& Marlière, L. D. P. (2006). Complementary/Alternative Medicine in Latin America: Use of Herbal Remedies among a Brazilian Metropolitan Area Population. Journal of Complementary and Integrative Medicine, 3(1), 1-12.

Brasil. (2016). Ministério da Saúde. Secretaria de Ciência, Tecnologia e Insumos Estratégicos. Departamento de Assistência Farmacêutica. Política e Programa Nacional de Plantas Medicinais e Fitoterápicos. - Brasília: Ministério da Saúde, 2016.

Brasil. (2015). Miniterio Da Saúde. RENAME. Relação Nacional de Medicamentos Essenciais - RENAME 2014. Ministério da Saúde. Brasília, DF, 9a edição.

Brasil. (2012). Ministério da Saúde. Secretaria de Atenção à Saúde. Departamento de Atenção Básica. Práticas integrativas e complementares: plantas medicinais e fitoterapia na Atenção $\quad$ Básica/Ministério https://bvsms.saude.gov.br/bvs/publicacoes/praticas_integrativas_complementares_plantas_medicinais_cab31.pdf

Brasil. (2011). Ministério da Saúde. Secretaria de Atenção à Saúde. Departamento de Atenção Básica. Coordenação Nacional de Práticas Integrativas e Complementares. Relatório de Gestão 2006/2010: Práticas Integrativas e Complementares no SUS. Brasília: Ministério da Saúde.

Brasil. (2010). Ministério da Saúde. Agência Nacional de Vigilância Sanitária. Resolução RDC nº 14, de 31 de março de 2010 . Dispõe sobre o registro de medicamentos fitoterápicos. Diário Oficial da União. 2010. 1(1): 7-85.

Brasil. (2009). Ministério da Saúde. RENISUS - Relação Nacional de Plantas Medicinais de Interesse ao SUS. Espécies vegetais DAF/SCTIE/MS - RENISUS - Agência Saúde. Brasília, DF, 03 mar. 2009.

Brasil. (2006a). Ministério da Saúde. Secretaria de Atenção à Saúde. Departamento de Atenção Básica. Política Nacional de Práticas Integrativas e Complementares no SUS, PNPIC, SUS. Brasília: Ministério da Saúde. 
Brasil. (2006b). MINISTÉRIO DA SAÚDE. Secretaria de Ciência, Tecnologia e Insumos Estratégicos Departamento de Assistência Farmacêutica e Insumos Estratégicos. A Fitoterapia no SUS e o Programa de Pesquisas de Plantas Medicinais da Central de Medicamentos. Brasília. 2006.

Brasil. (2004). Ministério da Saúde. Agência Nacional de Vigilância Sanitária. Resolução de Diretoria Colegiada no. 48 de 16 de março de 2004 aprova o regulamento técnico de medicamentos fitoterápico junto ao Sistema Nacional de Vigilância Sanitária. DOU. Diário Oficial da União, Poder Executivo, DF, Brasília, 2004.

Bruning, M. C. R., mosegui, G. B. G., \& Vianna, C. M. M. (2012). A utilização da fitoterapia e de plantas medicinais em unidades básicas de saúde nos municípios de Cascavel e Foz do Iguaçu - Paraná: a visão dos profissionais de saúde. Ciência \& Saúde Coletiva, 17(10), 2675-2685.

Carvalho A. C. B., Balbino, E. E., Maciel, A., \& Perfeito, J. P. S. (2008). Situação do registro de medicamentos fitoterápicos no Brasil. Revista Brasileira de Farmacognosia-Brazilian Journal of Pharmacognosy, 18(2), 314-319.

Carvalho, A. C. B. (2007). Aspectos da Legislação no controle dos medicamentos fitoterápicos. T\&C Amazônia, 11(5), 26-32.

Darroz, J. V., Fuso, L. C., Borges, N. M., \& Gomes, A. J. P. S. (2014). Utilização de fitoterápicos no tratamento de constipação intestinal. Arquivos de Ciências da Saúde UNIPAR, 18(2), 113-119.

Dias, E. C. M., Trevisan, D. D., Nagai, S. C., Ramos, N. A., \& Silva, E. M. (2017). Uso de fitoterápicos e potenciais riscos de interações medicamentosas: reflexões para prática segura. Revista Baiana de Saúde Pública, 41(2), 297-307.

Figueredo, C. A., Gurgel, I. G. D., \& Junior, G. D. G. (2014). A Política Nacional de Plantas Medicinais e Fitoterápicos: construção, perspectivas e desafios. Physis Revista de Saúde Coletiva, 24(2), 381-400.

Fontenele, R. P., Sousa, D. M. P., Carvalho, A. L. M., \& Oliveira, F. A. (2013). Fitoterapia na Atenção Básica: olhares dos gestores e profissionais da Estratégia Saúde da Família de Teresina (PI), Brasil. Ciência \& Saúde Coletiva, 18(8), 2385-2394.

Gadelha, C. S., Junior, V. M. P., Berreza, K. K. S., Maracajá, P. B., \& Martins, D. S. S. (2015). Utilização de medicamentos fitoterápicos e plantas medicinais em diferentes segmentos da sociedade. Revista Verde (Pombal -PB -Brasil), 10(3), 01-15

Hoeffner, R., Heck, R. M., Ceolin, T., Jardim, V. M. R., \& Barbieri, R. L. (2012). Plantas medicinais utilizadas para o alívio da dor pelos agricultores ecológicos do Sul do Brasil. Revista Eletrônica Enfermagem, 14(3), 596-602.

Ibiapina, W. V., Leitão, B. P., Batista, M. M., \& Pinto, D. S. (2014). Inserção da fitoterapia na atenção primária aos usuários do sus. Revista ciência em saúde nova esperança, $12(1), 58-68$.

Lombardo, M. (2021). Fitoterápicos na atenção básica de problemas gastrointestinais. Revista Ciências em Saúde, 6(1), 34-47.

Lucena, J. A. S., \& Guedes, J. P. M. (2020). Uso de fitoterápicos na prevenção e no tratamento da hipertensão arterial sistêmica. Revista Brasileira de Educação e Saúde, 10(1), 15-22.

Marmitt, D. J., Rempel, C., Goettrt, M. I., \& Silva, A. C. (2015). Plantas Medicinais da RENISUS Com Potencial Anti-inflamatório: Revisão Sistemática Em Três Bases de Dados Científicas. Revista Fitos, 9(2), 73-159

Mello, M. B., Rodriguez J. A., Morelli, H., \& Melo, S. F. (2019). A importância do uso de fitoterápicos no Sistema Único de Saúde. Revista Científica Multidisciplinar Núcleo do Conhecimento, 11(5), 118-131.

Moraes, 1. C., Alonso, A. M., \& Filho, E. C. O. (2011). Plantas medicinais no tratamento do câncer: uma breve revisão de literatura. Universitas: Ciências da Saúde, 9(1), 77-99.

Moreira, V. P., Peixoto, A. P., \& Marchionni, A. M. T. (2020). Uso de medicamentos fitoterápicos como opção anti-inflamátoria na odontologia. Journal of Dentistry \& Public Health, 11(2), 167-175.

Oliveira, A. B., Longhi, J. G., Andrade, C. A., Gomes, O. M., \& Dallormi, M. (2007). Brazilian phytotherapic regulamentation. universidade federal do paraná, 1(1), 1-13.

Pinha, A. G; Alves, I. F; Romanosque, J. Neto, M. J. (2019). O uso de plantas medicinais no município de três lagoas. Revista Saúde e Meio Ambiente, 9(3), 24-33.

Pinto, E. G., Cavalcante, F. S. A., \& Lima, R. A. (2020). A fitoterapia no tratamento de pele: um estudo bibliográfico. Biodiversidade, 19(3), 188-197.

Queiroz, M. S. (2000). O itinerário rumo às medicinas integrativas: uma análise em representações sociais de profissionais da saúde. Caderno de Saúde Pública, 16(1), 363-75.

Reinecken, J. L., Mendes, A. B., Maeques, A. G., \& Bennemann, R. M. (2018). Plantas medicinais potencialmente hipolipidêmicas. Enciclopédia biosfera, Centro Científico Conhecer, 15(28), 1289-1303.

Reis, F. C. (2018). Projeto de implementação do uso adequado da fitoterapia na unidade de saúde de águas férreas do município de são pedro dos ferros, minas gerais. Universidade federal de minas gerais, 1(1), 1-34.

Santos, D. S., Maciel, J. C., \& Dias, G. B. (2018). Principais plantas medicinais e fitoterapia na terapêutica de afecções estomacais: uma revisão bibliográfica. Health Diversity, 2(1), 86-90.

Santos, A. M. A., Miranda, M. G., Cardoso, F. T., Moraes, S. R., \& Avelar, K. E. S. (2013). Popular phytotherapy: past and present. Espacios, $34(11), 1-7$.

Santos, R. L., Guimaraes, G. P., Nobre, M. S. C., \& Portela, A. S. (2011). Análise sobre a fitoterapia como prática integrativa no Sistema Único de Saúde. Revista Brasileira de Plantas Medicinais, 13(4), 486-491. 
Research, Society and Development, v. 11, n. 1, e44611124643, 2022

(CC BY 4.0) | ISSN 2525-3409 | DOI: http://dx.doi.org/10.33448/rsd-v11i1.24643

Silva, R. Y. A. (2019). Estudo dos efeitos de espécies vegetais da relação nacional de plantas medicinais de interesse ao sus sobre o receptor pxr. 2019. 79f. Dissertação (Mestrado) - Pós-Graduação em Ciências Farmacêuticas. Faculdade de Ciências da Saúde, Universidade de Brasília.

Tomazzoni, M. I., Negrelle, R. R. B., \& Centa, M. L. (2006). Fototerapia Popular: A Busca Instrumental Enquanto Prática Terapêutica. Texto Contexto Enfermagem. 15(1), 21-115.

Vieira, A. R. R., \& Medeiros, P. R. M. S. (2019). A utilização de fitoterápicos no tratamento da obesidade. Revista Cientifica da Escola Estadual Saúde Pública Goiás "Cândido Santiago", 5(1), 44-57.

Zardeto-sabec, G., Jesus, R. A., Quemel, F. S., \& Zenaide, F. S. (2019). Medicinal plants as na alternative in the treatment of cancer. Brazilian Journal of Surgery and Clinical Research, 27(3), 75-80. 